\title{
Readmissions in adult patients following hospitalization for influenza: a nationwide cohort study
}

\author{
Srikanth Yandrapalli, Wilbert S. Aronow, William H. Frishman \\ Department of Medicine, New York Medical College at Westchester Medical Center, Valhalla, NY, USA \\ Contributions: (I) Conception and design: All authors; (II) Administrative support: None; (III) Provision of study materials or patients: None; (IV) \\ Collection and assembly of data: None; (V) Data analysis and interpretation: All authors; (VI) Manuscript writing: All authors; (VII) Final approval of \\ manuscript: All authors. \\ Correspondence to: Srikanth Yandrapalli, MD. Division of Internal Medicine, Westchester Medical Center, 100 Woods Road, Taylor Pavilion, Valhalla, \\ NY 10595, USA. Email: srikanth.yn@gmail.com.
}

\begin{abstract}
Background: Influenza epidemics are a major health care concern in the US. Influenza related complications can increase in-hospital complications, and readmissions following a hospitalization for influenza. We sought to determine the 30-day readmission rate, etiologies, outcomes, and healthcare burden of 30-day readmissions in adults hospitalized for influenza.
\end{abstract}

Methods: The 2014 US National Readmissions Database (NRD) was retrospectively analyzed to identify patients $\geq 18$ years of age hospitalized for influenza and discharged between January and November 2014 . We used this time frame as this was the most recent data available for analysis and included patients who had 30-day follow-up. Survey design based multivariable logistic regression models were used to identify factors associated with a 30-day readmission.

Results: Of the 46,117 patients who were hospitalized for influenza and survived to discharge, 4,721 (10.2\%) patients had 5,275 30-day readmissions, estimated to 11.4 readmissions per 100 patients. Non-influenza pneumonia was the most common etiology of 30-day readmissions (10.4\%) followed by sepsis (9.8\%). The median costs of readmissions were $\$ 8,538$ (IQR, \$5,053-15,262), which were significantly higher than the median costs of their index hospitalizations [\$7,863 (IQR, \$4,875-13,212); $\mathrm{P}<0.001$ ]. Around $6.5 \%$ of the patients died during a readmission.

Conclusions: Adult patients hospitalized for influenza had 11.4 30-day readmissions per 100 patients, most commonly for non-influenza pneumonia. Thirty-day readmissions were associated with higher costs of care and considerable mortality.

Keywords: Influenza; acute myocardial infarction (AMI); stroke; readmissions; pneumonia

Submitted Apr 20, 2018. Accepted for publication Jul 19, 2018.

doi: $10.21037 /$ atm.2018.07.18

View this article at: http://dx.doi.org/10.21037/atm.2018.07.18

\section{Introduction}

Influenza is a major public health problem in the US with significant variations in its annual incidence and public health burden (1). While the majority of influenza patients are managed in the ambulatory setting, influenza and related complications can result in hospitalizations $(1,2)$. The Center for Disease Control (CDC) reported an estimated minimum 140,000 and maximum 710,000 hospitalizations for influenza and related complications between years 2010 and 2016 in the US $(1,2)$. In the US in year 2003, influenza-related hospitalizations amounted to $\$ 6.0$ billion in costs, which were only $7 \%$ of the total economic burden of influenza during that year (3). Among adult patients hospitalized for influenza, more than twothirds have underlying medical conditions, most commonly cardiovascular (CV) disease, metabolic disorder, obesity, and chronic lung disease (4). Further, available data 
suggest an increased risk of adverse $\mathrm{CV}$ events like acute myocardial infarction (AMI) and ischemic cerebrovascular events (CVEs) in the acute phase following an influenza infection (5-14).

While such risk of increased adverse events can increase in-hospital complications, they can also cause readmissions in these patients in the acute phase following influenza infection. Readmissions are usually measured within 30 days because readmissions and deaths after a longer time period may be less influenced by the admitting condition and care gotten in the hospital and more to do with other complicating illnesses, patients' behavior, or care provided to patients after hospital discharge (15). Data are scarce regarding the etiology and burden of 30-day readmissions following a hospitalization for influenza. We sought to determine the 30-day readmission rates and associated factors, reasons for readmission and their healthcare burden, after hospitalization for either influenza-pneumonia or influenza-related organ system manifestations in adult patients.

\section{Methods}

\section{Data source}

In this retrospective observational study, data were collected from the publicly available 2014 US National Readmissions Database (NRD). The NRD is part of the Healthcare Cost and Utilization Project (HCUP) that is sponsored by the Agency for Healthcare Research and Quality (AHRQ) and is a database of all-payer hospital inpatient stays that can be used to generate national estimates of readmissions (16). The NRD is drawn from HCUP State Inpatient Databases which contain verified patient linkage numbers that can be used to track a person across readmissions while maintaining privacy. The NRD includes community hospitals and excludes rehabilitation or longterm acute care hospitals. Unweighted, the 2014 NRD contains data from approximately 15 million discharges from 22 partner states: Arkansas, California, Florida, Georgia, Hawaii, Iowa, Louisiana, Maryland, Massachusetts, Missouri, Nebraska, New Mexico, Nevada, New York, South Carolina, South Dakota, Tennessee, Utah, Virginia, Vermont, Washington, and Wisconsin. These states are geographically dispersed and account for $51.2 \%$ of the total US resident population and $49.3 \%$ of all hospitalizations. Weighted, it estimates roughly 35 million discharges (16).

The NRD was post-stratified by hospital characteristics (census region, urban/rural location, hospital teaching status, size of the hospital defined by the number of beds, and hospital control) and patient characteristics [sex and five age groups (0,1-17, 18-44, 45-64, and 65 and older)] (16). Discharge weights (variable DISCWT) were provided to obtain national estimates.

\section{Patient population and baseline characteristics}

The study sample included all adult patients ( $\geq 18$ years) who were discharged alive between January and November 2014 with a primary discharge diagnosis of influenza pneumonia or other influenza-related manifestations. These cases formed our index cohort. Patients discharged in December were excluded because the NRD design will not allow for 30-day follow-up of patients discharged in this month. International Classification of Diseases, Ninth Edition, Clinical Modification (ICD-9-CM) diagnosis codes 487 and 488 were used to identify influenza diagnoses. We chose data from 2014 for our study as these were the most recently available data which used ICD-9 codes throughout for coding diagnosis. If a patient had multiple hospitalizations for influenza during the study period, only the first hospitalization for influenza was included in the index cohort. We did not include hospitalizations with a secondary discharge diagnosis of influenza because such hospitalizations could include patients with previously diagnosed influenza, co-incidental influenza diagnosis, and also hospital-acquired influenza when admitted for other reasons.

Baseline patient characteristics identified in the index cohort were age, sex, primary expected payer, median household income, comorbidities (anemia, atrial fibrillation, cancer, chronic lung disease, coagulopathy, depression, drug abuse, dementia, diabetes mellitus, hypertension, hypothyroidism, fluid-electrolyte imbalances, liver disease, obesity, other neurological disorders, paralysis, psychoses, peripheral vascular disease, pulmonary circulation disorders, chronic renal failure, and heart failure), inhospital complications [septicemia and shock, AMI, acute kidney injury (AKI), ischemic stroke and transient cerebral ischemic events, gastrointestinal hemorrhage, and respiratory failure), type and day of admission, and discharge disposition. Hospital characters (location, bedsize, and teaching status) were also identified. Elixhauser comorbidity data were extracted from the NRD. ICD9-CM and the HCUP Clinical Classification Software 
(CCS) codes used to identify comorbidities and in-hospital complications are available in Table S1.

\section{Study outcomes}

The primary outcomes of this study were the 30-day readmission rate from the day of discharge, etiology for readmission, and the factors associated with 30-day readmissions. Secondary outcomes were comparison of length of stay (LOS) and costs of care between index hospitalization and readmission, and all-cause in-hospital mortality during 30 -day readmissions only because all patients in our index cohort survived to discharge. LOS and costs of hospitalization were identified in both index cases and readmission, whereas, all-cause inpatient mortality was identified in 30-day readmissions. Methodology described by the HCUP to identify readmissions was used to identify 30-day readmissions (16). Total number of 30-day readmissions and the number of index-patients readmitted within 30-day were identified and expressed as proportion of the index hospitalizations. Time to readmission was calculated as the duration between index-hospital discharge and the first day of hospital readmission.

Etiologies of readmissions were recognized by identifying the ICD-9-CM diagnosis codes and the HCUP CCS codes of the primary discharge diagnosis of each individual readmission (available in Table S2). Causes of readmissions were classified on the basis of organ system involvement into respiratory, $\mathrm{CV}$, infection-related, neurovascular, peripheral vascular, hematologic and cancer related, gastrointestinal, renal and fluid-electrolyte disorders, endocrine and metabolic, neuropsychiatric and substance abuse-related, musculoskeletal and injury related, symptom related (fever of unknown origin, malaise, abdominal pain, nausea/vomiting), complication of devicegraft-implant, or others. Respiratory causes included noninfluenza pneumonia and empyema, influenza, obstructive lung disease, pleural disease, respiratory failure, aspiration pneumonitis, and others. CV causes included heart failure, arrhythmias and conduction disorders, AMI, hypertension, coronary artery disease, pulmonary embolism, syncope, and chest pain. Infection-related etiologies included sepsis, intestinal infections, skin infections, urinary tract infections (UTIs), and others.

To identify costs of hospitalization, hospital reported charges (variable TOTCHG) were multiplied with the costto-charge ratios developed and reported by the AHRQ.

\section{Statistical analysis}

All statistical analyses were performed using IBM SPSS Statistics 25.0 (IBM Corp., Armonk, NY, USA). Weighted data were used for all statistical analyses. Categorical variables are expressed as percentages, and continuous variables as mean \pm standard deviation $(\mathrm{SD})$. Because the distributions of LOS and total hospital costs were skewed to the right, median [interquartile range (IQR)] were also reported. Baseline patient characters, comorbidities and complications, and hospital characteristics were compared between patients with and without 30-day readmission using Pearson $\chi^{2}$ test for categorical variables and student $t$-test for continuous variables. Nonparametric MannWhitney $\mathrm{U}$ test was used to compare medians between patient groups. Since the NRD has a complex semirandom multistage sampling survey design, survey design based multivariable logistic regression model [designed to test for $95 \%$ confidence intervals (CIs) and a two-sided $\mathrm{P}$ value threshold of $<0.05$ ] was designed to identify factors associated with 30-day readmissions. The regression model included all the variables which achieved a $\mathrm{P}$ value $<0.2$ in unadjusted analysis. In the NRD, patients' aged $\geq 90$ years were reported as 90-year-old. This can affect the results when age (in years) is used as a covariate in statistical analysis. Hence, patients were sub grouped into three age categories (18-44, 45-64, and $\geq 65$ years). LOS of index hospitalizations was included in the regression model as a categorical variable divided into 2 groups based on median LOS cut-off. Results of the logistic regression analyses were reported as odds ratios (ORs) and $95 \%$ CIs.

Around $\leq 1 \%$ data were missing for the variables primary payer status, median household income, discharge disposition, and type of admission (elective versus nonelective). Missing values were replaced with the dominant categories. Total hospital charge data were missing for around $1 \%$ index-hospitalizations and $0.9 \%$ of the 30 -day readmission. Reported cost data were for the remainder of the cases.

\section{Results}

\section{Baseline characteristics of the study cohort and in-hospital complications}

The index-study cohort included 46,117 adult patients who had influenza hospitalization meeting our index-case criteria and survived to discharge. These patients had a 
mean age \pm SD of $61.1 \pm 17.9$ years and $53.6 \%$ were female. Of these, 22,457 (48.7\%) were primarily hospitalized for influenza-pneumonia, 22,375 (48.5\%) for influenza-related respiratory symptoms, and 1,285 (2.8\%) for other organsystem manifestations of influenza. Overall, around $44.5 \%$ patients were elderly with age $\geq 65$ years. Baseline patient demographics, comorbidities, in-hospital complications, and hospital characteristics for the overall index-study cohort are presented in Table 1. More patients (31.1\%) were in the lowest median income quartile $(\$ 1-39,999)$ group and around two-thirds had either Medicare or Medicaid health insurance. Hypertension, chronic pulmonary disease, diabetes mellitus, fluid-electrolyte disturbances, and anemia were the most prevalent comorbidities. While respiratory failure occurred in $22.1 \%$ of index hospitalizations, AKI complicated $12.8 \%$, an $\mathrm{AMI}$ in $1.2 \%$, and ischemic CVE in $0.3 \%$. More patients in the index-cohort were hospitalized in large-sized urban-based teaching hospitals. Median LOS was relatively short at 3 days [IQR, 2-6 days] and mean $\mathrm{LOS} \pm \mathrm{SD}$ was $5.1 \pm 6.9$ days. The total costs of index hospitalizations were $\$ 489.9$ million, mean $\$ 10,729 \pm 22,255$ and median \$6,082 (IQR, \$3,921-10,220).

\section{Thirty-day readmissions and characteristics of index hospitalizations by readmission status}

Of the 46,117 index-group patients, 4,721 (10.2\%) patients had 5,275 30-day readmissions. This estimates to 11.4 readmissions per 100 patients within a 30-day period from discharge. Baseline patient demographics, comorbidities, in-hospital complications, and hospital characters for the index cases with and without a readmission are presented in Table 1.

Patients who had a 30-day readmission were generally older $(63.9 \pm 17.5$ years $)$ compared to those without a 30 -day readmission $(60.7 \pm 17.9$ years; $\mathrm{P}=0.02)$, more likely to be insured by Medicare/Medicaid (74.9\% vs. $64.6 \%$; $\mathrm{P}<0.001$ ), and more likely to have a higher burden of comorbidities and in-hospital complications. Ischemic CVEs $(0.6 \%$ vs. $0.3 \%$; $\mathrm{P}=0.005)$, AMI $(2.1 \%$ vs. $1.2 \%$; $\mathrm{P}<0.001)$, and gastrointestinal bleeding $(1.4 \%$ vs. $0.7 \%$; $\mathrm{P}<0.001)$ occurred almost two-times the rate in patients who had a 30-day readmission compared to those who did not. Patients who had a 30-day readmission had a longer median initial LOS [4 days (IQR, 3-7 days) vs. 3 days (IQR, 2-5 days); $\mathrm{P}<0.001]$, more likely to be discharged to a nursing home or other medical facility $(20.4 \%$ vs. $12.3 \%$; $\mathrm{P}<0.001)$ and had higher median index hospitalization costs
[\$7,863 (IQR \$4,875-13,212) vs. \$5,946 (\$3,830-9,804); $\mathrm{P}<0.001]$.

\section{Factors associated with a 30-day readmission}

In multivariable logistic regression analysis results presented in Table 2, presence of chronic pulmonary disease (OR 1.15; 95\% CI, 1.03-1.27), drug abuse (OR 1.53; 95\% CI, 1.181.99), liver disease (OR 1.47; 95\% CI, 1.12-1.92), chronic renal failure (OR 1.42; 95\% CI, 1.23-1.64), heart failure (OR 1.45; 95\% CI, 1.25-1.69), AKI (OR 1.24; 95\% CI, $1.07-1.44$ ), anemia (OR 1.32; 95\% CI, 1.17-1.50), cancer (OR 1.93; 95\% CI, 1.56-2.39), and sepsis (OR 1.31; 95\% CI, 1.01-1.68) were associated with a 30-day readmission. A longer LOS >3 days (OR 1.48; 95\% CI, 1.31-1.67), discharge to nursing home/other medical facility (OR 1.23; 95\% CI, 1.07-1.44), and admission to a teaching hospital (OR 1.15; 95\% CI, 1.03-1.29) also predicted a 30-day readmission.

\section{Timing and causes of 30-day readmissions}

Of the 5,275 readmissions, $45.8 \%$ were within 10 days from discharge, $29.3 \%$ between days $11-20$, and $24.8 \%$ between days 21-30. Median time to readmission was 12 days (IQR, 5-20 days). Etiologies of readmissions were presented in Table 3. When stratified according to organ-system involvement, most common causes of 30 -day readmission were respiratory $(31.2 \%)$, infectious (15.8\%), CV (14.9\%), gastrointestinal (7.6\%), and hematologic-cancer related (4.9\%). Diagnosis wise, noninfluenza pneumonia was the most common etiology of 30 -day readmissions $(10.4 \%)$, followed by sepsis $(9.8 \%)$, obstructive lung disease (8.0\%), heart failure (6.2\%), AKI and UTIs at $2.3 \%$ each. While a microbiological diagnosis was not made in most non-influenza pneumonias (78.4\%), methicillin-resistant Staphylococcus aureus (MRSA) was the most frequently identified organism $(4.8 \%)$ in the rest. Similarly, around $81 \%$ of the sepsis-related admissions did not have a microbiologic diagnosis, but when an organism was identified, it was more often gram-positive (12\%; $5 \%$ streptococcal and 4\% MRSA).

\section{LOS, costs, and mortality related to 30-day readmissions}

Overall, $6.5 \%$ of the patients readmitted within 30-days from discharge died during a readmission. Mortality was highest when the readmission etiology was infectious, 
Table 1 Baseline characteristics, comorbidities, in-hospital complication, and outcomes of patients in the index-cohort with and without a 30-day readmission

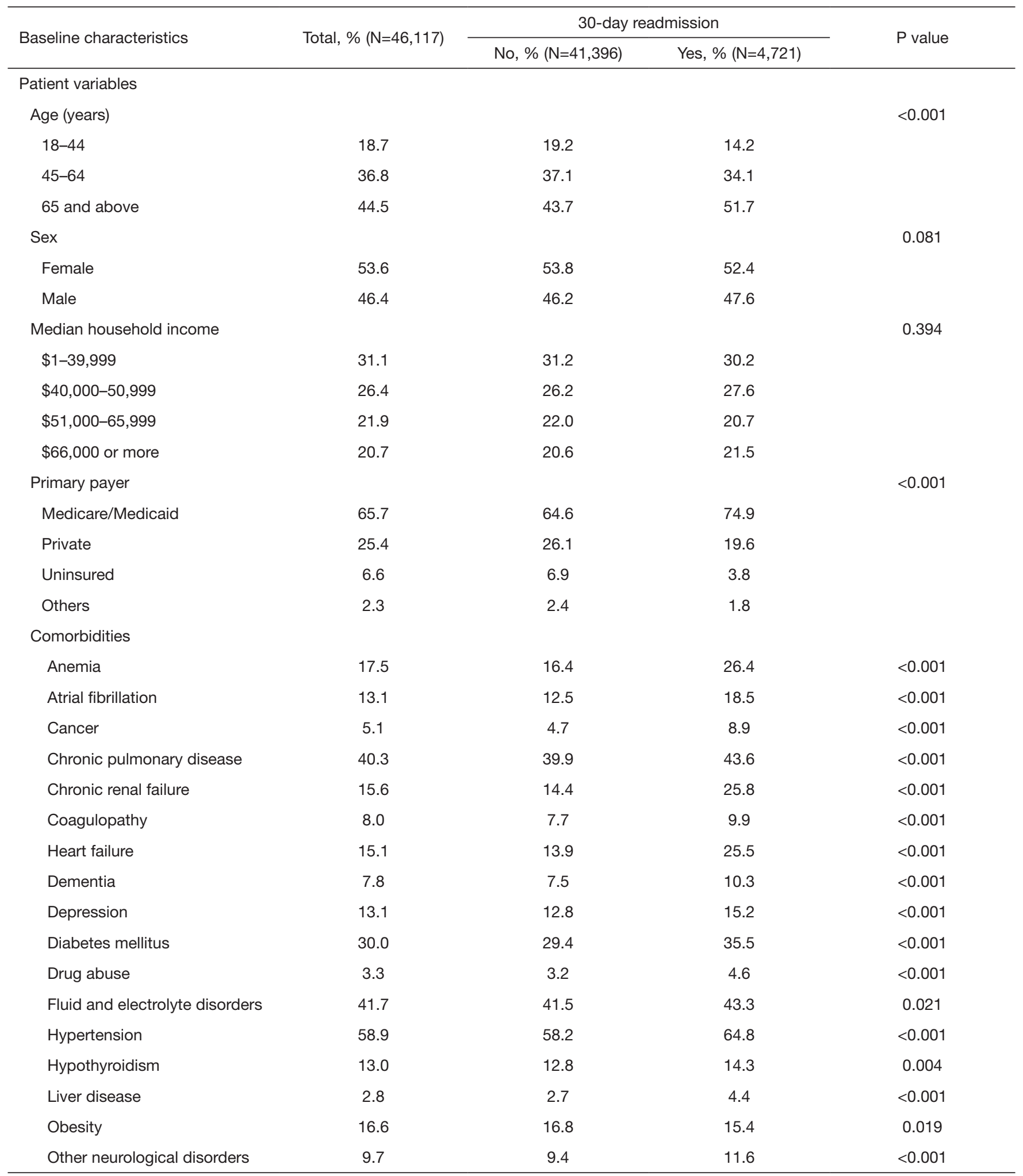

Table 1 (continued) 
Table 1 (continued)

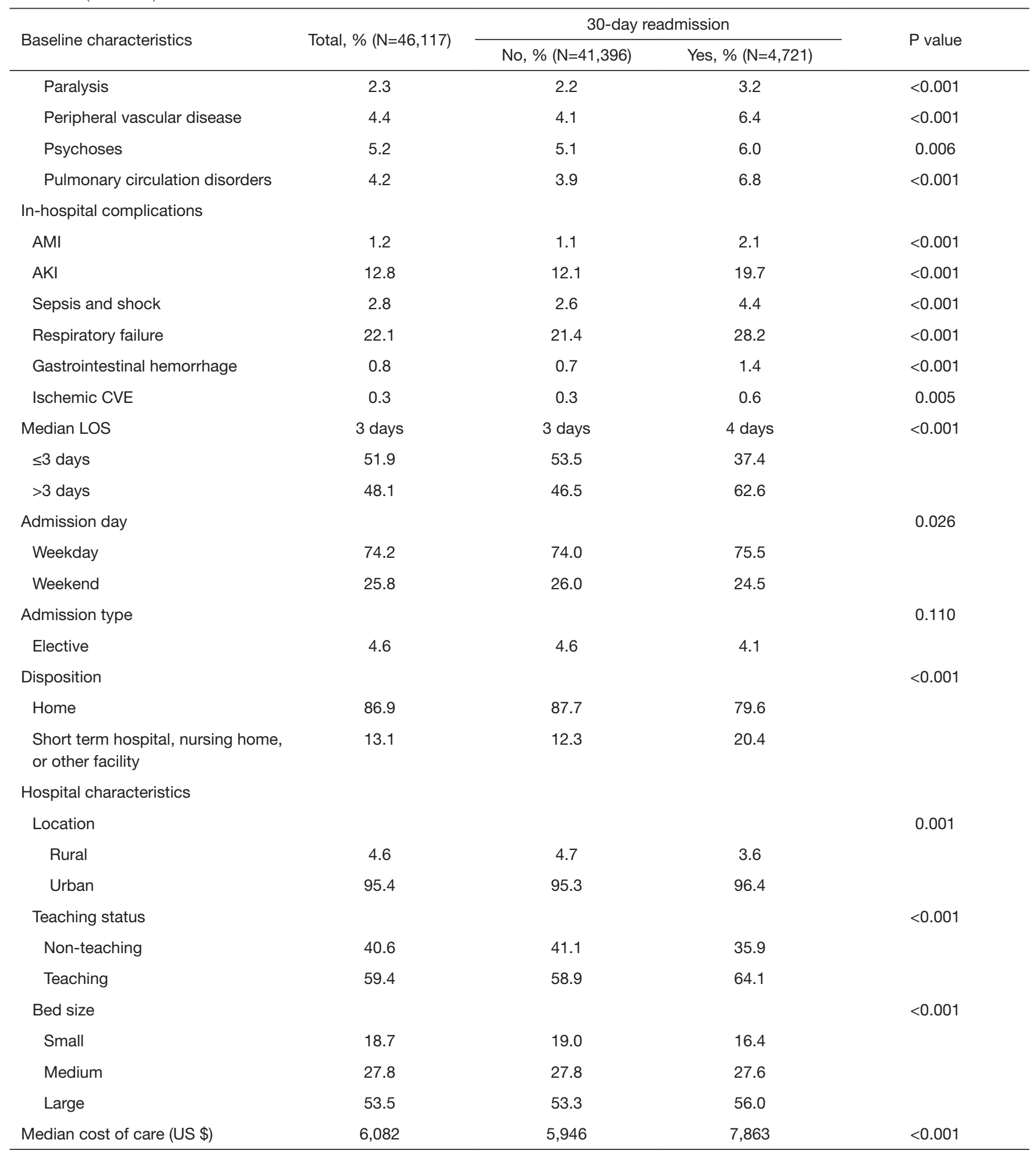

AMI, acute myocardial infarction; AKI, acute kidney injury; LOS, length of stay; CVE, cerebrovascular event. 
Table 2 Results of the multivariable regression analysis to identify factors associated with increased 30-day readmissions

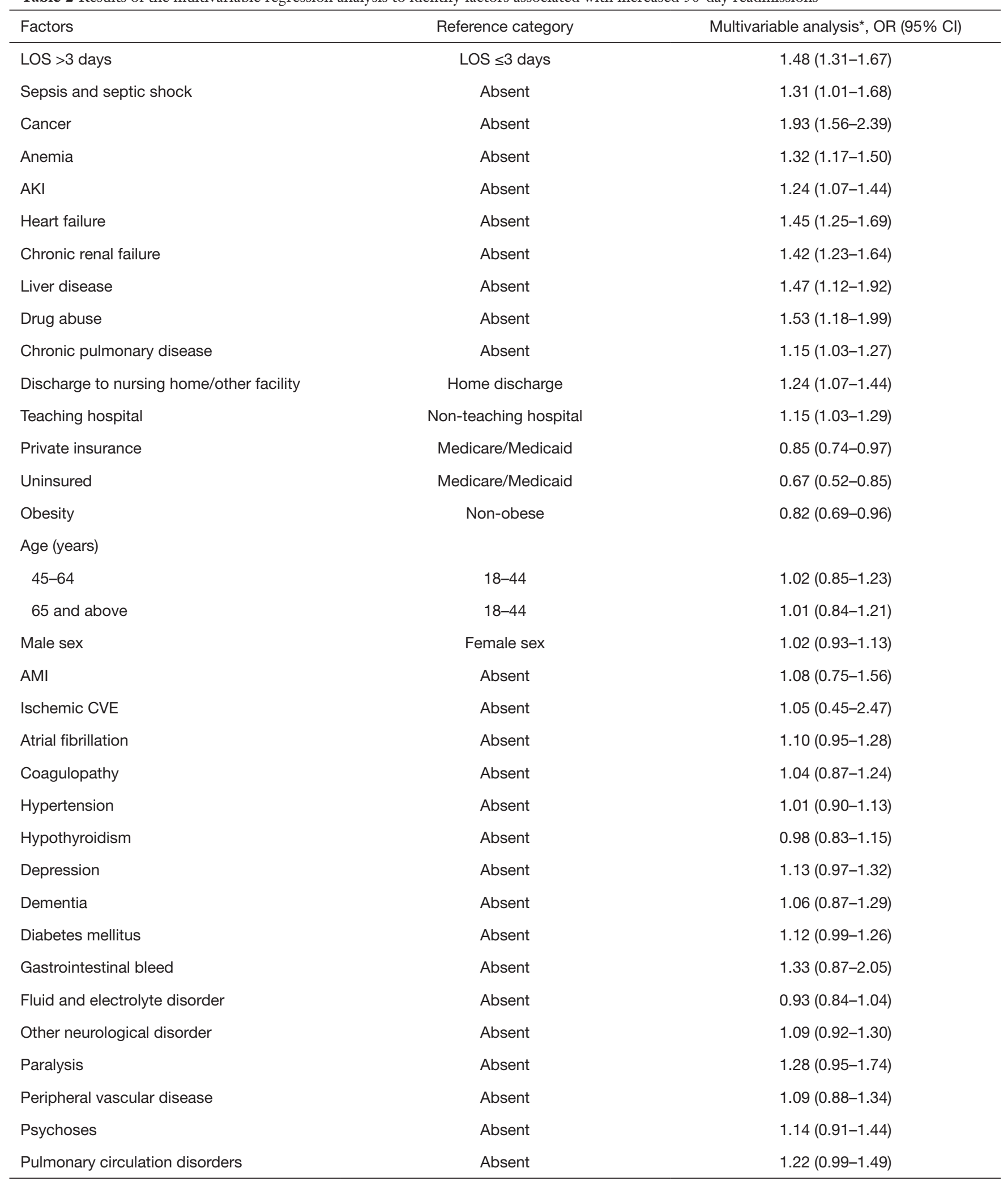

Table 2 (continued) 
Table 2 (continued)

\begin{tabular}{lcc}
\hline Factors & Reference category & Multivariable analysis*, OR (95\% Cl) \\
\hline Respiratory failure & Absent & $1.12(0.98-1.28)$ \\
Elective admission & Non-elective admission & $0.95(0.72-1.26)$ \\
Weekend admission & Weekday admission & $0.89(0.79-1.01)$ \\
Rural-based hospital & Urban-based hospital & $1.02(0.74-1.39)$ \\
Bed size of hospital & & $1.15(0.99-1.33)$ \\
Large & Small & $1.16(0.99-1.37)$ \\
\hline Medium & Small & \\
\hline
\end{tabular}

", survey design based multivariable logistic regression model designed to test for 95\% confidence intervals and a two-sided $\mathrm{P}$ value of < 0.05 . OR, odds ratio; Cl, confidence intervals; LOS, length of stay; AKI, acute kidney injury; AMI, acute myocardial infarction; CVE, cerebrovascular event.

Table 3 System based and disease specific etiologies of 30-day readmission after hospitalization for influenza

\begin{tabular}{lc}
\hline Etiology of readmission & $\begin{array}{c}\text { 30-day readmissions } \\
(\mathrm{N}=5,275, \%)\end{array}$ \\
\hline Respiratory & $1,643(31.1)$ \\
Non-influenza pneumonia and empyema & $549(10.4)$ \\
Influenza & $190(3.6)$ \\
Chronic obstructive lung disease and & $422(8.0)$ \\
asthma & \\
Aspiration pneumonitis & $127(2.4)$ \\
Pleural disease & $32(0.6)$ \\
Respiratory failure & $223(4.2)$ \\
Other respiratory tract disease & $100(1.9)$ \\
Cardiovascular & $784(14.9)$ \\
Heart failure & $327(6.2)$ \\
Conduction disease and arrhythmias & $127(2.4)$ \\
Acute myocardial infarction & $63(1.2)$ \\
Coronary artery disease & $37(0.7)$ \\
Hypertension & $63(1.2)$ \\
Pulmonary embolism & $84(1.6)$ \\
Syncope & $32(0.6)$ \\
Nonspecific chest pain & $42(0.8)$ \\
Infection-related and septicemia & $516(9.8)$ \\
\hline & $11.7)$ \\
Untestinal infection & $2.3)$ \\
\hline
\end{tabular}

Table 3 (continued)
Table 3 (continued)

\begin{tabular}{|c|c|}
\hline Etiology of readmission & $\begin{array}{c}\text { 30-day readmissions } \\
(\mathrm{N}=5,275, \%)\end{array}$ \\
\hline Skin and soft-tissue infection & $60(1.1)$ \\
\hline Others & $42(1.0)$ \\
\hline Neurovascular & $104(2.0)$ \\
\hline Ischemic cerebrovascular events & $59(1.1)$ \\
\hline Peripheral vascular & $91(1.7)$ \\
\hline Endocrine and metabolic & $119(2.3)$ \\
\hline Gastrointestinal & $402(7.6)$ \\
\hline Gastrointestinal hemorrhage & $106(2.0)$ \\
\hline $\begin{array}{l}\text { Renal diseases and fluid/electrolyte } \\
\text { imbalance }\end{array}$ & $243(4.6)$ \\
\hline Acute kidney injury & $119(2.3)$ \\
\hline Fluid and electrolyte disorder & $96(1.8)$ \\
\hline Neuropsychiatric and substance-related & $242(4.6)$ \\
\hline Musculoskeletal and injury related & $229(4.3)$ \\
\hline Symptom-related & $109(2.0)$ \\
\hline Fever of unknown origin & $38(0.7)$ \\
\hline Nausea/vomiting & $22(\leq 0.5)$ \\
\hline Abdominal pain & $28(\leq 0.5)$ \\
\hline Malaise and fatigue & $15(\leq 0.5)$ \\
\hline Device, implant, graft complications & $129(2.4)$ \\
\hline Others & $90(1.7)$ \\
\hline
\end{tabular}


reaching a high of $21.5 \%$ in patient admitted for septicemia. Median LOS was 4 days (IQR, 3-8 days) with a mean of $6.5 \pm 7.6$ days. Total costs associated with 30 -day readmissions were around $\$ 77.2$ million during the study period. Median readmission costs were $\$ 8,538$ (IQR, $\$ 5,053-15,262$ ) and mean costs were $\$ 14,772 \pm 23,348$. The median LOS of the readmissions (4 days) was significantly higher than the median LOS of all index admissions ( 3 days; $\mathrm{P}<0.001$ ), but was similar to the median LOS of the index cases which had a readmission ( 4 vs. 4 days; $\mathrm{P}=0.77$ ). The median costs of readmissions $(\$ 8,538)$ were significantly higher compared to median costs of all index cases $(\$ 6,082 ; \mathrm{P}<0.001)$ and index cases with a 30-day readmission $(\$ 7,863 ; \mathrm{P}<0.001)$.

\section{Discussion}

In this retrospective observational study of a large group of patients who were representative of the US population and were primarily hospitalized for influenza in 2014, a substantial number of patients were readmitted within 30 -days from discharge, with an estimated $11.4 \%$ readmission rate.

Respiratory system diseases and infections were the most common etiologies for a 30-day readmission. Influenza infection increases the risk of secondary pneumonias' particularly bacterial (17). In our study, non-influenza pneumonia was the most common cause of 30-day readmissions. A microbiological diagnosis was not made in around four-fifths of the cases in our study, but the most commonly identified organism was MRSA in $5 \%$. During influenza epidemics, both Streptococcus pneumonia and Staphylococcus aureus (more recently MRSA) were identified as common causes of bacterial pneumonias (17).

Prior studies have reported an increased risk of AMI and stroke in the acute phase following influenza (5-7,9$11,13,14,18,19)$. In our study, AMI and ischemic CVEs had incident rates of $1.2 \%$ and $0.3 \%$ respectively and were infrequent causes of 30-day readmissions (between 1-2\%). However, it must be noted that we only included patients who survived to discharge during an index hospitalization in our analysis and the hence did not fully capture incidence rate of AMI or ischemic CVEs in all adults hospitalized for influenza. Influenza infection has not been clearly implicated to cause heart failure and obstructive lung disease exacerbations (20-22). In our study, hospitalizations for heart failure, chronic obstructive pulmonary disease, and asthma were important causes 30-day readmissions.

In our study, increasing age categories and differences in sex did not influence 30-day readmission risk. Anemia, cancer, heart failure, chronic lung disease, liver disease, and chronic renal failure independently predicted a 30-day readmission. These conditions increase the comorbid disease burden in patients and can result in multiple hospitalizations. AMI and ischemic CVEs, which occurred infrequently in our index group patients, were not associated with a 30-day readmission. Interestingly, LOS more than 3 days was independently associated with a readmission. A longer LOS might suggest a sicker patient but a LOS higher than the median predicted a readmission after accounting for common in-hospital complications and comorbidities. Patients had a higher 30-day readmission risk when discharged to a nursing home or other medical facility. Patients discharged to a nursing home should have more supervision of care to help reduce hospitalizations. However, patients typically discharged to a nursing facility are older with a higher comorbidity burden and severity of illness associated with increased readmissions (23). Early and appropriate discharge planning to reduce LOS and to aim for home-discharge with adequate support might help reduce the readmission rate in this patient group.

Readmissions also represented a significant health-care and economic burden. Among patients hospitalized within 30 days from discharge, $6.5 \%$ died. More patients died when the cause of readmission was infectious. Appropriate infection control strategies during index hospitalization and early identification and treatment of septicemia should be emphasized to decrease this high mortality rate. The economic burden of readmissions was higher than the index admissions despite having a similar LOS. These findings suggest higher health-care resource utilization during readmissions.

The strengths of our study are utilization of a large nationally representative administrative database which was collected using validated methods to reduce selection bias. Using validated methodology, we were able to determine the 30-day readmission rates, identify the causes and predictors of readmissions, and determine their economic impact in the real-world patients hospitalized for influenza (16).

Our study has important limitations. Firstly, administrative databases lack clinical and laboratory variables which can more accurately determine duration of infection and patient status. It is not known if influenza was diagnosed based on clinical symptoms or laboratory testing in our study. Secondly, using billing codes to identify causes of hospitalizations and in-hospital complications can result in misclassification 
bias. Thirdly, data on anti-influenza medication use and vaccination was not available. We were unable to test the association of immunization and anti-viral treatment on readmission risk. Fourthly, our sample included influenza patients across 2 different seasons (January-November) and excluded patients discharged in December. The design of the NRD, which is a yearly database, limited our ability to perform analysis bases on flu-season. We could not adjust for viral-strain and seasonal based differences in readmission rates. Lastly, the NRD does not report out of hospital and emergency room mortality data, which could influence the readmission rates.

\section{Conclusions}

In conclusion, adult patients hospitalized for influenza had 11.4 30-day readmissions per 100 patients, most commonly for non-influenza pneumonia and other infections. Thirtyday readmissions were associated with significant healthcare impact with higher costs of care and considerable mortality.

\section{Acknowledgements}

None.

\section{Footnote}

Conflicts of Interest: The authors have no conflicts of interest to declare.

\section{References}

1. Disease Burden of Influenza. Seasonal Influenza (Flu). Available online: https://www.cdc.gov/flu/about/disease/ burden.htm

2. Estimated Influenza Illnesses, Medical Visits, Hospitalizations, and Deaths Averted by Vaccination in the United States. Seasonal Influenza (Flu). Available online: https://www.cdc.gov/flu/about/disease/2015-16.htm

3. Molinari NA, Ortega-Sanchez IR, Messonnier ML, et al. The annual impact of seasonal influenza in the US: measuring disease burden and costs. Vaccine 2007;25:5086-96.

4. Weekly U.S. Influenza Surveillance Report. Seasonal Influenza (Flu). Available online: https://www.cdc.gov/flu/ weekly/index.htm

5. Barnes M, Heywood AE, Mahimbo A, et al. Acute myocardial infarction and influenza: a meta-analysis of case-control studies. Heart 2015;101:1738-47.

6. Estabragh ZR, Mamas MA. The cardiovascular manifestations of influenza: a systematic review. Int $\mathrm{J}$ Cardiol 2013;167:2397-403.

7. Madjid M, Naghavi M, Litovsky S, et al. Influenza and cardiovascular disease: a new opportunity for prevention and the need for further studies. Circulation 2003;108:2730-6.

8. Flu and Heart Disease \& Stroke. https://www.cdc.gov/flu/ heartdisease/index.htm

9. Urbanek C, Palm F, Grau AJ. Influenza and stroke risk: a key target not to be missed? Infect Disord Drug Targets 2010;10:122-31.

10. Warren-Gash C, Smeeth L, Hayward AC. Influenza as a trigger for acute myocardial infarction or death from cardiovascular disease: a systematic review. Lancet Infect Dis 2009;9:601-10.

11. Toschke AM, Heuschmann PU, Wood O, et al. Temporal relationship between influenza infections and subsequent first-ever stroke incidence. Age Ageing 2009;38:100-3.

12. Nguyen JL, Yang W, Ito K, et al. Seasonal Influenza Infections and Cardiovascular Disease Mortality. JAMA Cardiol 2016;1:274-81.

13. Muhammad S, Haasbach E, Kotchourko M, et al. Influenza virus infection aggravates stroke outcome. Stroke 2011;42:783-91.

14. Kwong JC, Schwartz KL, Campitelli MA, et al. Acute Myocardial Infarction after Laboratory-Confirmed Influenza Infection. N Engl J Med 2018;378:345-53.

15. The Official U.S. Government Site for Medicare. Available online: https://www.medicare.gov/hospitalcompare/ Data/30-day-measures.html

16. The Hcup Nationwide Readmissions Database (NRD), 2014. Available online: https://www.hcup-us.ahrq.gov/db/ nation/nrd/NRD_Introduction_2014.jsp

17. Metersky ML, Masterton RG, Lode H, et al. Epidemiology, microbiology, and treatment considerations for bacterial pneumonia complicating influenza. Int J Infect Dis 2012;16:e321-31.

18. Clar C, Oseni Z, Flowers N, et al. Influenza vaccines for preventing cardiovascular disease. Cochrane Database Syst Rev 2015;5:CD005050.

19. Udell JA, Zawi R, Bhatt DL, et al. Association between influenza vaccination and cardiovascular outcomes in highrisk patients: a meta-analysis. JAMA 2013;310:1711-20.

20. Kurai D, Saraya T, Ishii H, et al. Virus-induced exacerbations in asthma and COPD. Front Microbiol 2013;4. doi:10.3389/fmicb.2013.00293 
21. Mallia P, Johnston SL. Influenza infection and COPD. Int J Chron Obstruct Pulmon Dis 2007;2:55-64.

22. Sandoval C, Walter SD, Krueger P, et al. Risk of hospitalization during influenza season among a cohort of patients with congestive heart failure. Epidemiol Infect
2007;135:574-582.

23. Kripalani S, Theobald CN, Anctil B, et al. Reducing hospital readmission: current strategies and future directions. Annu Rev Med 2014;65:471-85.
Cite this article as: Yandrapalli S, Aronow WS, Frishman WH. Readmissions in adult patients following hospitalization for influenza: a nationwide cohort study. Ann Transl Med 2018;6(16):318. doi: 10.21037/atm.2018.07.18 
Supplementary

Table S1 ICD-9CM and the CCS codes used to identify primary discharge diagnoses, baseline comorbidities, and in-hospital complications

\begin{tabular}{|c|c|c|}
\hline Diagnosis & ICD-9-CM codes & CCS codes \\
\hline Influenza & & 123 \\
\hline Influenza-pneumonia & $487.0,488.01,488.11,488.81$ & \\
\hline Other respiratory manifestations & $487.1,488.02,488.12,488.82$ & \\
\hline Other manifestations & $487.8,488.09,488.19,488.89$ & \\
\hline Dementia & $\begin{array}{c}290 . x x, 294.1 x, 294.2 x, 331.0,331.1 x \\
331.82,331.89\end{array}$ & \\
\hline Atrial fibrillation & 427.31 & \\
\hline \multicolumn{3}{|l|}{ In-hospital complications } \\
\hline Acute myocardial infarction & $410 . x 1,411.1$ & \\
\hline Acute kidney injury & $584 . x$ & \\
\hline $\begin{array}{l}\text { Ischemic stroke and transient } \\
\text { cerebral ischemia }\end{array}$ & $433 . x 1$ & 112 \\
\hline
\end{tabular}


Table S2 ICD-9CM and the CCS codes in the primary discharge diagnosis position used to identify causes of 30-day readmissions

\begin{tabular}{|c|}
\hline Organ system/diagnosis \\
\hline Respiratory \\
\hline Pneumonia and empyema \\
\hline Influenza \\
\hline COPD and asthma \\
\hline Aspiration pneumonitis \\
\hline Pleural disease \\
\hline Respiratory failure \\
\hline Other respiratory tract disease \\
\hline Cardiovascular \\
\hline Heart failure \\
\hline Conduction disease and arrhythmia \\
\hline AMI \\
\hline CAD \\
\hline Hypertension \\
\hline Pulmonary embolism \\
\hline Syncope \\
\hline Nonspecific chest pain \\
\hline Neurovascular \\
\hline Ischemic stroke and transient cerebral ischemia \\
\hline Peripheral vascular \\
\hline Hematologic and cancer-related \\
\hline Endocrine and metabolic \\
\hline Gastrointestinal \\
\hline GIB \\
\hline
\end{tabular}

Renal diseases and fluid/electrolyte imbalance

AKI

CCS codes
$56,122-134$
122
123

ICD-9-CM codes

127, 128

129

510.9, 511.0, 511.89, 511.9, 512.0, 512.89

$518.8 x$

$125,126,133,134$

96-108, 245

108

$402.91,404.91,404.93,788.51$

$105,106,107$

100

101

414.04

98,99

$415.1 x$

245

102

109-113

112

$434.11,434.91$

114-121

$14,15,16,18,19,24,33,35,38,39,40,42,44$, $45,47,59-63$

$50,51,58$

138-141, 143-155

153

$55,157,158,160-163$

584. $x$

55

$81,83,84,85,93,95,651,653,654,657,659$, 660,661

54, 203-205, 207, 210-212, 226, 229-234, 238-

244

Symptoms

Fever of unknown origin

246

Nausea/vomiting

250

Abdominal pain

251

Malaise and fatigue

252

Device, implant, graft complications

Others

237

$89,92,136,137,165-167$,

$171,175,182,187,189,193,199,253,254,257,259$

$x$, the ICD-9CM subcode; ICD-9CM, International Classification of Diseases, Ninth Edition, Clinical Modification; CCS, Clinical Classification Software; COPD, chronic obstructive lung disease; AMI, acute myocardial infarction; CAD, coronary artery disease; UTI, urinary tract infection; AKI, acute kidney injury. 